\title{
Paraplegia due to Spinal Epidermoid Cyst Rupture at Asthma Attack
}

\author{
Kweon Young Kim, MD, Jung Hun Kang, MD, Dae Woo Choi, MD, Min Hong Lee, MD, Jae Hyouk Jang, MD
}

Department of Rehabilitation Medicine, Chosun University Hospital, Gwangju, Korea

Spinal epidermoid cyst is less than $1 \%$ of the entire spinal cord tumor and a rare tumor. It is a slowly proliferating benign tumor and can be a result of either congenital or acquired factors. In particular, reports of acute paraplegia due to spinal epidermoid cyst rupture are very rare. Since authors experienced paraplegia resulting from congenital spinal epidermoid cyst rupture during an asthma attack, it is reported with a review of literature.

Keywords Epidermoid cyst, Asthma, Paraplegia

\section{INTRODUCTION}

Spinal epidermoid cyst is a very rare, slowly progressing benign tumor, and it can be developed due to either congenital or acquired factors [1]. According to its development mechanism, epidermoid cyst can be classified as acquired tumor for those with a medical history of spinal tap and congenital tumor related to abnormal invagination of the ectoderm at the time of neural canal closing is closed during 3-5 weeks of embryonic stage [2]. Spinal epidermoid cyst is reported to be found in less than $1 \%$ of spinal cord tumors for adults and in less than $3 \%$ for children, while $40 \%$ of spinal epidermoid cyst is reported to be related to the repetition of spinal tap [3]. In rare cases, symptoms present due to rupture, but most reported cas-

Received June 13, 2012; Accepted August 7, 2012

Corresponding author: Jae Hyouk Jang

Department of Rehabilitation Medicine, Chosun University Hospital, 365 Pilmun-daero, Dong-gu, Gwangju 501-717, Korea

Tel: +82-62-220-3153, Fax: +82-62-223-0245, E-mail: pooh3800@naver. com

(c) This is an open-access article distributed under the terms of the Creative Commons Attribution Non-Commercial License (http://creativecommons. org/licenses/by-nc/3.0) which permits unrestricted noncommercial use, distribution, and reproduction in any medium, provided the original work is properly cited.

Copyright $\odot 2013$ by Korean Academy of Rehabilitation Medicine es are caused by encephalomeningitis due to the outflow of substance from the cystoma or to neurologic symptoms due to inflammatory response. In particular, reports of acute paraplegia due to cystoma rupture are very rare, while most reports are of cases with gradual paralysis and spinal cord injury or neurologic symptoms after surgery. Moreover, most studies or reports about spinal epidermoid cyst are done by neurosurgeons or orthopedists, who performed the surgeries, and radiologists, who made the diagnosis in terms of radiology. Therefore, it was difficult to find studies of spinal epidermoid cyst with an approach in the field of rehabilitation medicine. As our rehabilitation department has experienced crural palsy due to rupture of a congenital spinal epidermoid cyst during an asthma attack in a healthy male patient, we report this case study with literature review.

\section{CASE REPORT}

This 22-year-old male patient intermittently suffered from hay fever in summer with symptoms of cough and dyspnea, however as his symptoms were minor, he did not undergo any special check-up, evaluation or treatment. When he was studying in college, he had symptoms of gradual backache and radiating pain in both lower 
limbs, but as symptoms were minor, he did not seek any examination. In November 2011, he started his military service as a conscripted policeman and experienced several rounds of difficulty in breathing and received conservative treatment. In January 2012, he showed severe dyspnea, coughing, chest pain, and muscular weakness in the lower limbs. After the pulmonary function test in a private hospital, he was diagnosed with asthma, so he was hospitalized and treated with a $\beta$-agonist and steroid injection. After 2 days, the symptoms continued so he was transferred to the emergency room of our hospital. After treatment in our hospital, his symptoms improved compared to the results of the private hospital's pulmonary function test, as shown by the forced expiratory volume of subnormal 1 second (FEV1) from $1.13 \mathrm{~L} \mathrm{(32 \%}$ of predicted value) to $3.50 \mathrm{~L}$ ( $78 \%$ of predicted value), the FEV1/forced vital capacity (FVC) from $46 \%$ to $77 \%$ and forced expiratory flow (FEF; range, $25 \%-75 \%$ ) from $11 \%$ to $67 \%$. His symptoms of dyspnea and chest pain gradually improved, but he continued to show muscular weakness in both lower limbs. The muscle tone of both upper limbs was within the normal level, but the muscle tone of both lower limbs was accelerated, showing increased deep tendon reflex in the knee and ankle joints, while exhibiting ankle clonus for both ankles. A neurogenic lesion in the upper part was suspected. According to manual muscle test, muscular strength of both upper limbs was normal, and there was no difference in muscular strength between both sides of lower limbs. However, the flexor/ extensor muscles of the hip and knee joints were $1 / 5$, and the flexor muscle of the instep/sole had been decreased to $0 / 5$. Moreover, spasticity of both lower limbs was level 1 for ankle dorsiflexion on the modified Ashworth Scale.

Blood test during his hospitalization showed a potassium level of $3.8 \mathrm{mEq} / \mathrm{L}$; thyroid function test did not show any unusual findings with $\mathrm{T} 3$ of $57.7 \mathrm{ng} / \mathrm{dL}$, thyroidstimulating hormone of $1.15 \mu \mathrm{IU} / \mathrm{mL}$, and free $\mathrm{T} 4$ of 1.38 $\mathrm{ng} / \mathrm{dL}$. He did not have a high fever, headache or meningism symptoms including Kernig's sign and Brudzinski's sign, so lumbar puncture test was not performed. From lumbar magnetic resonance imaging (MRI) which was done on the third day of hospitalization in our hospital, a severe stricture and compression were observed from L2 to S1 due to synechia and a cystic mass of posterior intraspinal space (Fig. 1). After 1 day of test, neurosurgical department of our hospital diagnosed him with lumbar cord damage, and then conducted emergency decompression surgery. Since a space between L2 and S1 was filled with a ruptured epidural cystoma, dead skin cells, and cholesterol type substances, they compressed severely the dura mater and nerves. Adhesion in the dura mater and the back spinal cord was also observed, however as it was located outside the dura mater, compressing substances were comparably easily and perfectly removed. Histological examination of the removed substances confirmed a ruptured epidermoid cyst together with simple squamous cells, dead skin cell components, and dystrophic calcification (Fig. 2). As the patient did
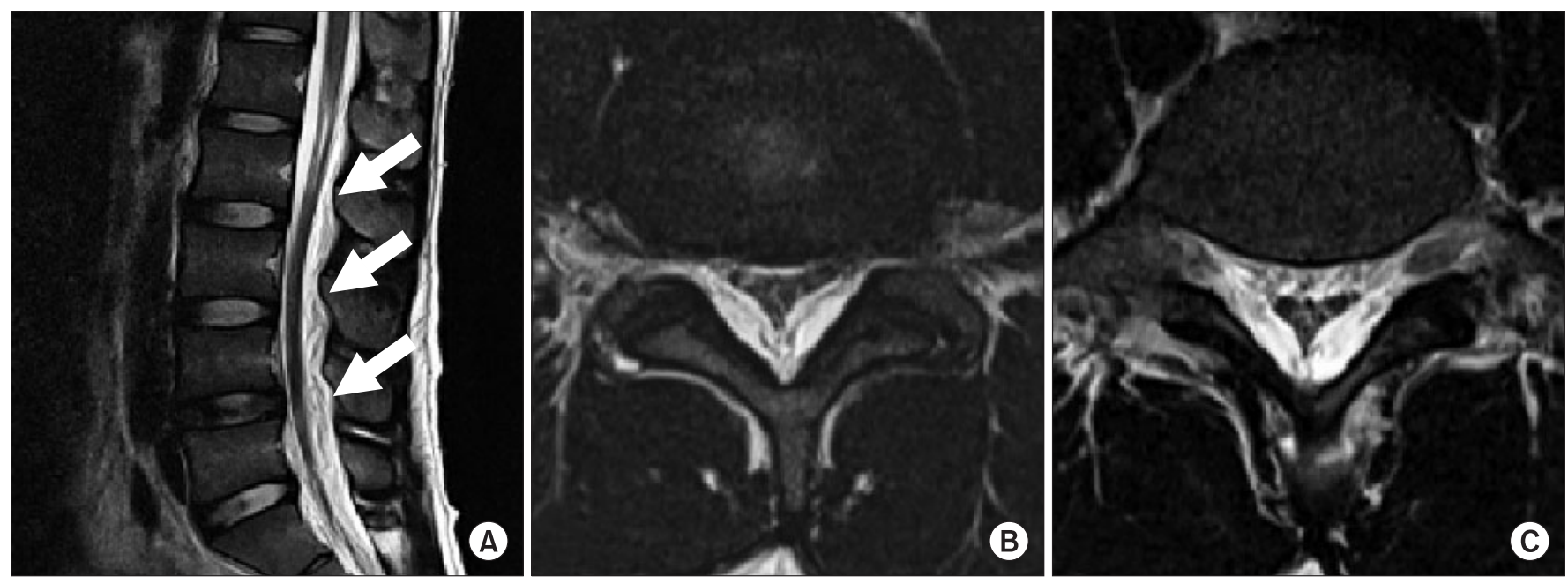

Fig. 1. Magnetic resonance imaging of the lumbar spine. Sagittal T2-weighted image shows an intraspinal cystic mass with adhesion extending from L2 to S1 (arrow). The signal of the mass is inhomogeneous (A). Axial T2-weighted image shows an intraspinal heterogeneous mass causing severe spinal stenosis at the L2 (B) and L3 levels (C). 
not undergo back surgery or rachicentesis, he was diagnosed with a congenital epidermoid cyst.

Seven days since the operation, the patient was transferred to our rehabilitation department; when he was transferred, manual muscle test showed no big difference in the muscular force of both lower limbs, while the flexor/extensor muscles of the hip and knee joints was $2 / 5$ and the flexor muscle of instep/sole was $1 / 5$. He was recovering, but, except for changing positions, sitting by himself and moving around in the wheel chair, his physi-

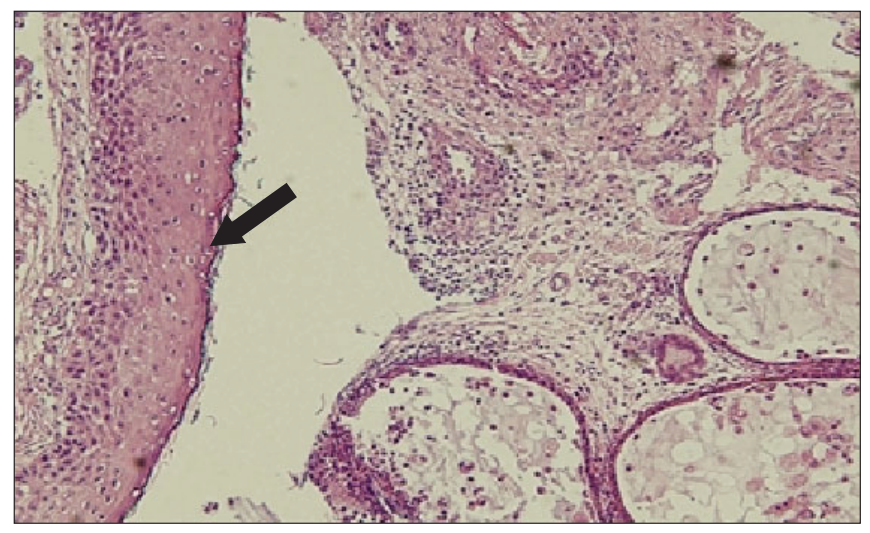

Fig. 2. Photomicrograph of the tumor mass near the cyst wall (H\&E staining, $\times 100)$. The cyst containing keratin is lined by benign keratinizing squamous epithelium (arrow). The stroma abundant skin appendages. cal strength became weaker such that he needed help from other people to perform everyday activities.

When he was transferred, the Functional Independence Measure (FIM) was 94, the Korean version of Medified Barthel Index (K-MBI) was 56 and the Spinal Cord Independence Measure (SCIM) was 58. According to results of his neurologic examination, he showed hypesthesia and muscle weakness from the dermatomere of L2, while anal contraction could be done by himself. Based on the motor nerve and sensory nerve, a neurologic damage part was L1 in paresis and could be classified as $\mathrm{C}$ in the American Spinal Injury Association (ASIA) Impairment Scale. From the initial period of hospitalization, a urethral catheter was inserted due to exacerbation of his entire body, so it was difficult to recognize urination symptoms, yet bulbocavernous reflex existed and was under drug treatment due to symptoms of slight constipation. Before the transfer, he was able to urinate by himself after removing the catheter, but exhibited residual urine and intermittent urinary frequency symptoms. After drug treatment, his symptoms improved. Electrodiagnosis was taken 1 week after the transfer (after 3 weeks since the operation); conduction test of the motor and sensory nerves showed normal latency and amplitude in both lower limbs, while the latency of both sides' H-reflexes was delayed. By conducting somatosensory

Table 1. Electrophysiologic findings

\begin{tabular}{|c|c|c|c|c|c|c|}
\hline \multirow[b]{2}{*}{ Nerve } & \multicolumn{3}{|c|}{ Right } & \multicolumn{3}{|c|}{ Left } \\
\hline & OL (ms) & $\begin{array}{c}\text { Amplitude } \\
(\mathrm{mV} / \mu \mathrm{V})\end{array}$ & $\mathrm{CV}(\mathrm{m} / \mathrm{s})$ & OL (ms) & $\begin{array}{c}\text { Amplitude } \\
(\mathrm{mV} / \mu \mathrm{V})\end{array}$ & $\mathrm{CV}(\mathrm{m} / \mathrm{s})$ \\
\hline \multicolumn{7}{|l|}{ SNAP } \\
\hline Sural & 2.8 & 28.0 & - & 2.8 & 26.5 & - \\
\hline Superficial peroneal & 2.8 & 29.0 & - & 2.8 & 24.7 & - \\
\hline \multicolumn{7}{|l|}{ CMAP } \\
\hline Peroneal (EDB) & 4.0 & 10.8 & 43 & 4.1 & 11.5 & 44 \\
\hline Tibial (AH) & 4.3 & 15.9 & 43 & 4.5 & 16.6 & 42 \\
\hline \multicolumn{7}{|l|}{ SEPs (P37) } \\
\hline Posterior tibial nerve & 46.9 & - & - & 46.0 & - & - \\
\hline \multicolumn{7}{|l|}{ MEPs } \\
\hline Tibial (AH) & 47.3 & - & - & 46.7 & - & - \\
\hline \multicolumn{7}{|l|}{ H-reflex } \\
\hline Tibial (GCM) & 32.4 & - & - & 32.6 & - & - \\
\hline
\end{tabular}

OL, onset latency; $\mathrm{CV}$, conduction velocity; SNAP, sensory nerve action potential; CMAP, compound muscle action potentials; EDB, extensor digitorum brevis muscle; AH, abductor hallucis muscle; SEPs, somatosensory evoked potentials; MEPs, motor evoked potentials; H-reflex, Hoffmann's reflex; GCM, gastrocnemius muscle. 
evoked potential of both tibial nerves and motor evoked potential at both abductor hallucis muscles by stimulating the head, we could observe a delay in the transcranial area (Table 1). In the needle electromyographic test, we observed a high level of abnormal spontaneous activities in the paravertebral muscles and lower limb muscles of that particular segment, demonstrating complications of conus medullaris syndrome and radiculopathy of the lumbosacral area (Table 2). Due to improved symptoms, the patient declined further examination, so tests for pu-

Table 2. Results of initial needle electromyography

\begin{tabular}{|c|c|c|c|c|}
\hline \multirow{2}{*}{ Muscle } & \multicolumn{2}{|c|}{ Abnormal spontaneous activity } & \multirow{2}{*}{ MUAP } & \multirow{2}{*}{ Recruitment pattern } \\
\hline & Fibrillation & PSW & & \\
\hline \multicolumn{5}{|l|}{ Right } \\
\hline Tibialis anterior & $1+$ & $1+$ & Long, poly & Discrete \\
\hline Peroneus longus & $1+$ & $1+$ & Long, poly & Discrete \\
\hline Extensor halluces longus & $1+$ & $1+$ & Long, poly & Discrete \\
\hline Gastrocnemius & $2+$ & $2+$ & Long, poly & Discrete \\
\hline Vastus medialis & $1+$ & $1+$ & Long, poly & Discrete \\
\hline Gluteus medius & $2+$ & $2+$ & Long, poly & Discrete \\
\hline Gluteus maximus & $2+$ & $2+$ & Long, poly & Discrete \\
\hline Lumbar paraspinalis & $3+$ & $3+$ & & \\
\hline \multicolumn{5}{|l|}{ Left } \\
\hline Tibialis anterior & $1+$ & $1+$ & Long, poly & Discrete \\
\hline Peroneus longus & $1+$ & $1+$ & Long, poly & Discrete \\
\hline Extensor halluces longus & $1+$ & $1+$ & Long, poly & Discrete \\
\hline Gastrocnemius & $1+$ & $1+$ & Long, poly & Discrete \\
\hline Vastus medialis & $1+$ & $1+$ & Long, poly & Discrete \\
\hline Gluteus medius & $2+$ & $2+$ & Long, poly & Discrete \\
\hline Gluteus maximus & $2+$ & $2+$ & Long, poly & Discrete \\
\hline Lumbar paraspinalis & $3+$ & $3+$ & & \\
\hline
\end{tabular}

PSW, positive sharp wave; MUAP, motor unit action potential; Long, long duration; poly, polyphasic motor unit action potentials.

Table 3. Comparison of ASIA muscle grades and motor scores before and after rehabilitation treatment

\begin{tabular}{|c|c|c|c|c|}
\hline \multirow{2}{*}{ Key muscles } & \multicolumn{2}{|c|}{ Before } & \multicolumn{2}{|c|}{ After } \\
\hline & Right & Left & Right & Left \\
\hline Elbow flexors & 5 & 5 & 5 & 5 \\
\hline Wrist extensors & 5 & 5 & 5 & 5 \\
\hline Elbow extensors & 5 & 5 & 5 & 5 \\
\hline Finger flexors (distal phalanx of middle finger) & 5 & 5 & 5 & 5 \\
\hline Finger abductors (little finger) & 5 & 5 & 5 & 5 \\
\hline Hip flexors & 2 & 2 & 4 & 4 \\
\hline Knee extensors & 2 & 2 & 4 & 4 \\
\hline Ankle dorsiflexors & 1 & 1 & 4 & 4 \\
\hline Long toe extensors & 1 & 1 & 3 & 3 \\
\hline Ankle plantar flexors & 1 & 1 & 3 & 3 \\
\hline Total ASIA motor score & & & & \\
\hline
\end{tabular}

ASIA, the American Spinal Injury Association. 
dendal somatosensory evoked potentials and bulbocavernosus reflex latency were not done.

During the hospitalization period in our hospital, the patient continued to exercise to strengthen the muscular power of the lower limbs through stimulating the functional nerve roots, standing up and gait training. Three months after the operation, the results of the manual muscle test showed the hip joint flexor, knee joint extensor and instep flexor muscles as $4 / 5$, while the hip joint extensor, and knee joint flexor and sole flexor muscles as $3 / 5$. The patient was able to walk a short distance on the flatland using a walker (Table 3). Before discharge, he scored 113 for FIM, 87 for K-MBI and 86 for SCIM. Currently, he is hospitalized for the second time for continual rehabilitation treatment and is under continual ambulatory observation by our department. He can now walk on the flatland using a quadripod cane.

\section{DISCUSSION}

Spinal epidermoid cyst is a very rare tumor found in less than $1 \%$ of spinal cord tumors and is classified as congenital or acquired cysts according to its development causes. In particular, congenital spinal epidermoid cyst is related to abnormal invagination of the ectoderm when the neural canal is closed during 3-5 weeks of embryonic stage; in such cases, it exists from birth and very slowly proliferates as the cells of the cystoma de-epithelize and then decompose as dead skin cells. Therefore, patients normally do not show any symptoms until adolescence or adulthood [4]. Cystic contents made during exfoliation of cystic epithelium arrangement and cystoma wall can calcificate, which will exacerbate the symptoms. Symptoms are similar to the conditions of general spinal cord tumor, such as muscular weakness, radiating pain, allesthesia, back pain, and more. For the present case, the patient was diagnosed with a congenital spinal epidermoid cyst since he did not have a medical history of repetitive spinal tap or myelomeningocele surgery but showed accompanying symptoms like diastematomyelia, congenital dermal sinus, myelomeningocele, and vertebral body. Congenital spinal epidermoid cyst can be found anywhere in the epidural, medulla subdural, and medulla areas, however, it is mostly found in the thoracic vertebrae area, especially between $\mathrm{T} 5$ and $\mathrm{T} 8$ and in the intradural-extramedullary area. On the other hand, an acquired spinal epidermoid cyst, especially if it is related to spinal tap, often develops underneath the arachnoid membrane of the lumbosacral area.

Congenital spinal epidermoid cyst develops very rarely, yet, sometimes, there are reports of various symptoms due to rupture. Nateghian et al. [5] reported a case of a 16-year-old boy suffering from a headache, fever and backache due to a medulla subdural spinal epidermoid cyst after lifting weights. Dobre et al. [6] announced a case of chemical encephalomeningitis in a 53-year-old female due to a spinal epidermoid cyst from voluntary rupture. Munshi et al. [7] reported a case of lower limb paralysis in a 3-year-old boy due to rupture of an epidermoid cyst in the medulla lumbar area after falling over as he was walking. They suggested bruises from the fall, violent cough and sneezing as causes of spinal epidermoid cyst rupture, which contributed to the increase of abdominal and spinal pressure. Moreover, they claimed that crural palsy develops from an inflammatory response due to substances in the cystoma when it is ruptured.

For the present case, the congenital spinal epidermoid cyst which was developed in the epidural area was ruptured from an increase of abdominal and spinal pressure from hard coughing and dyspnea due to asthma attacks. As a result, spinal cord compression occurred and finally caused acute crural palsy [8]. Spinal pressure can be directly increased during the Valsalva maneuver or sneeze and cough, while abdominal muscles work as expiratory muscles to move the ribs downward when patient coughs, resulting in increased abdominal pressure. During this process, spinal pressure increases due to congestion of blood in the epidural venous plexus around the spinal dura mater at the same time-thus, causing rupture. For cases of intradural epispinal or medulla subdural spinal epidermoid cyst, cystic inclusion can cause aseptic meningitis or inflammatory response when it inflows into the spine, plus, most of these types of case show strong adhesion of cystic capsule and nervous tissue, which can leave severe neurologic disability after surgery [9]. In the present case, the ruptured cyst was located in the epidural area, so there was adhesion between the dura mater and back spinal wall, but the prognosis of the surgery was better than that for intraduralextramedullary or medulla subdural spinal epidermoid cyst as it was relatively easy to be removed. Nonetheless, recovery after the surgery was slower compared to other 
similar cases, since early treatment was focused on emergency treatment in the private hospital, emergency room and the internal medicine department and did not recognize lower limb paralysis while the patient himself could not be aware of other symptoms due to his severe asthma attacks. As a result, emergency decompression operation was conducted after 6 days since the outbreak of symptoms, and the recovery period became longer because of delayed treatment.

For asthma patients, Hopkins syndrome or the chronic use of steroids can be considered as causes of crural palsy. However, this patient had allergic symptoms and was not diagnosed with asthma, plus, he was never on steroid treatment. Since Hopkins syndrome, which usually develops in children and is a rare disease causing weakened muscles and decreased muscle tone without hypesthesia after a few days of asthma attacks, has a bad prognosis, it could be excluded for this case.

Epidermoid cyst shows various signal intensities in MRI; normally, T1-weighted images have low signal intensity, while T2-weighted images have high signal intensity and are observed as non-uniform patterns. Even in this case, because of the special characteristics of the cyst including dead skin cell substances and scattering of cholesterol, the T2-weighted image showed a heterogeneous aspect with low signal intensity. However, as epidermoid cyst can show different MRI results depending on its chemical status, we should keep in mind that it may not show specific aspects.

Spinal epidermoid cyst is very rare and since its exhibition of symptoms is slow, thus making early detection in clinics difficult. However, if it is ruptured due to hard coughing, injury, bruise from a fall and sneezing, all of which cause increased spinal and abdominal pressure, it can lead to neurological disability and encephalomeningitis. Therefore, when a spinal lump is found, accurate and immediate evaluation and diagnosis are required. As we can see from this case study, failure of early diagnosis and treatment can delay the recovery or prognosis, thus, it will be necessary to conduct rehabilitation evaluation together with early detection and neurological evalua- tion for spinal cyst or lump for neurological disability. Furthermore, as accurate diagnosis, appropriate excision and enough rehabilitation treatment right after the detection of the disease will be able to maximize the functional recovery of the patient for quick return to his/her daily life, it is considered that the department of rehabilitation medicine should pay more attention to this disease.

\section{CONFLICT OF INTEREST}

No potential conflict of interest relevant to this article was reported.

\section{REFERENCES}

1. Manno NJ, Uihlein A, Kernohan JW. Intraspinal epidermoids. J Neurosurg 1962;19:754-65.

2. Russel DS, Rubistein LJ. Pathology of tumors of the nervous system. 5th ed. London: Edward Arnold; 1989.

3. Baba H, Wada M, Tanaka Y, Imura S, Tomita K. Intraspinal epidermoid after lumbar puncture. Int Orthop 1994;18:116-8.

4. Lee JH, Shin JJ, Hwang YS, Kim TH, Shin HS, Park SK. Intramedullary epidermoid cyst in thoracolumbar junction: a case report. Korean J Spine 2008;5:219-24.

5. Nateghian A, Mehta V, Robinson JL. Headache, fever and back pain in a 16-year-old boy. Can J Infect Dis 2004;15:53-4.

6. Dobre MC, Smoker WR, Moritani T, Kirby P. Spontaneously ruptured intraspinal epidermoid cyst causing chemical meningitis. J Clin Neurosci 2012;19:587-9.

7. Munshi A, Talapatra K, Ramadwar M, Jalali R. Spinal epidermoid cyst with sudden onset of paraplegia. J Cancer Res Ther 2009;5:290-2.

8. Williams B. On the pathogenesis of syringomyelia: a review. J R Soc Med 1980;73:798-806.

9. Penisson-Besnier I, Guy G, Gandon Y. Intramedullary epidermoid cyst evaluated by computed tomographic scan and magnetic resonance imaging: case report. Neurosurgery 1989;25:955-9. 